\title{
A Simulation Approach to Semiconductor Scheduling Problem in Micro- Lithographic Process
}

\author{
Shoaib Shafique \\ School of Biochemistry and Biotechnology, University of the Punjab Lahore, Pakistan \\ shoaibshafique39@gmail.com \\ 0000-0002-2299-5489
}

\begin{tabular}{|c|c|}
\hline Article History & Abstract \\
\hline $\begin{array}{l}\text { Article Submission } \\
04 \text { October } 2021 \\
\text { Revised Submission } \\
16 \text { November } 2021 \\
\text { Article Accepted } \\
18 \text { December } 2021 \\
\text { Article Published } \\
20 \text { January } 2022\end{array}$ & $\begin{array}{l}\text { In this write up, a scheduling issue and FDC in photolithographic process of } \\
\text { semiconductor manufacturing are thought of. By and large, the photolithographic } \\
\text { progression is viewed as the most significant processes since it might influence the } \\
\text { creation profitability because of its different mechanical traits. Truly, the } \\
\text { photolithography hardware comprises of three fundamental parts which are intended } \\
\text { to process various sorts of items as a machine for universally useful. Be that as it } \\
\text { may, in current semiconductor creation system, a similar kind of item is processed so } \\
\text { as to diminish formula change time brought about by changing veils in scanner } \\
\text { module. Also, FDC can quickly distinguish strange circumstance of activity machine, } \\
\text { in order to improve the yield rate. Along these lines, in this examination, the multi- } \\
\text { item creation case with various plans is considered in micro-lithographic process in } \\
\text { regards to scheduling issue, and a FDC system is built up that comprising of fluffy } \\
\text { deduction system and decision tree to screen and break down warming bend of } \\
\text { delicate prepare in micro-lithographic process. } \\
\text { Keywords: Semiconductor Manufacturing, Photolithography Process, Scheduling, } \\
\text { FDC system, decision tree }\end{array}$ \\
\hline
\end{tabular}

\section{Introduction}

As we probably are aware, semiconductor deploying procedure is an extremely perplexing and requires extravagant developed hardware. For a considerable length of time, numerous specialists have concentrated on the financially savvy utilization of gear of semiconductor manufacturing process. As consequences of the investigates, numerous surprising techniques for operational upgrades just as mechanical advances have been grown, for example, ideal scheduling calculations which can augment certain profitability measures. Normally, various kinds of items require distinctive creation processes just as various creation environment, that is, diverse handing out times and progression arrangements, and furthermore the measures of requests required from clients may vary from item to item. Micro-lithographic procedure in semiconductor industrialized system can fabricate various kinds of items with foreordained arrangement with prompt machine inactive time or holding up time. Along these lines, it is important to build up an effective creation plan which can boost the general manufacturing plant efficiency. In this manner, in this examination, we assess exhibitions of various information protocols of parcels and wafers in micro-lithography gear with considering 10 item creation plans as appeared in Table 1 where M1-M8 are modules and P1-P10 are items.

In semiconductor engineering, utensils cost of micro-lithographic process includes $30 \%$ of the supreme assembling charge that has the most significant degree when all is said in done. Photolithography is the procedure to make a structured wafer with progresses. This assessment revolves around progression of a FDC framework. FDC is specific from traditional frameworks. Traditional procedures are separated frameworks whereas FDC can subsequently screen each complexity between set worth and veritable estimation of machine. As showed by data, it will instantly separate equipment preparing ability to keep from further disorders. With FDC, weird condition of procedure limit can be found instantly, and a short time later be unwound in the right second to lessen cost achieved by blocked creation. 
Table 1: surge recipes of Products in micro-lithography

\begin{tabular}{|l|l|l|l|l|l|l|l|l|l|l|}
\hline Parameters & P1 & P2 & P3 & P4 & P5 & P6 & P7 & P8 & P9 & P10 \\
\hline M1 & 4 & 1 & 5 & 3 & 0 & 2 & 3 & 4 & 0 & 5 \\
\hline M2 & 2 & 2 & 3 & 0 & 1 & 3 & 2 & 2 & 0 & 3 \\
\hline M3 & 1 & 4 & 0 & 0 & 0 & 2 & 6 & 0 & 0 & 0 \\
\hline M4 & 0 & 3 & 0 & 0 & 1 & 1 & 4 & 0 & 2 & 2 \\
\hline M5 & 2 & 0 & 2 & 5 & 1 & 0 & 0 & 3 & 3 & 0 \\
\hline M6 & 3 & 0 & 1 & 3 & 2 & 2 & 1 & 1 & 1 & 0 \\
\hline M7 & 1 & 2 & 2 & 1 & 2 & 0 & 2 & 0 & 1 & 1 \\
\hline M8 & 0 & 1 & 1 & 0 & 5 & 0 & 1 & 0 & 4 & 1 \\
\hline
\end{tabular}

This investigation presents a FDC framework on the assessment of warming twist in fragile warmth step during micro-lithographic procedure to propel yield velocity. There are duo purposes in this assessment: one is the revelation anomalistic warming twists subsequently, using the area framework made by this investigation to dismember and to distinguish warming twists. Another aim is describing anomalistic warming curves with rule base created. This investigation in like manner settled a FDC framework on warming twist of sensitive warmth in photolithography region subject to fleecy methods of reasoning and choice tree. Since cushy speculation has a fair effort, we pick soft theory to perceive warming twists.

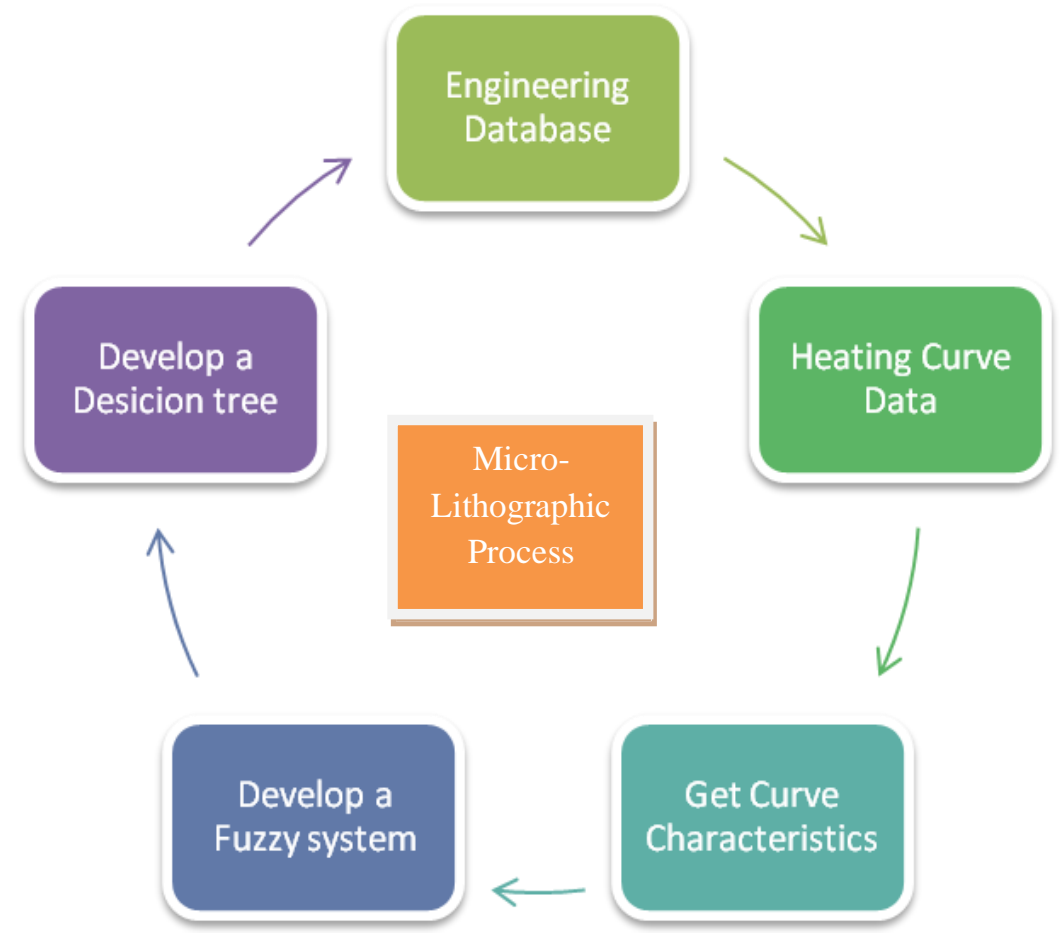

Figure 1. FDC System Procedures

\section{Reference}

[1] AJ. Pasadyn and T.F. Edgar, "Observability and State Estimation for Multiple Product Control in Semiconductor Manufacturing," IEEE, vol. 18, pp. 592, 2005.

[2] Park, H., Rhee, S., and Kim, D. (2001) A fuzzy pattern recognition-based system for monitoring laser weld quality, Measurement Science and Technology, Vol. 12, pp.1318-1324.

[3] W.J.Wang, S.F.Lee,and T.C.Chian (2001) Fuzzy control for the intersection's traffic light near the exit ramp of a freeway, Automatic Control Conference,Da-Shing,Taiwan, ROC. 